\title{
LONG-PERIODIC TRANSVERSE OSCILLATIONS OF CORONAL LOOPS AND MODULATIONS OF SOLAR MICROWAVE RADIATION
}

\author{
M. L. Khodachenko*, A. G. Kislyakov ${ }^{\dagger}$, K. G Kislyakova ${ }^{\dagger}$, \\ T. V. Zaqarashvili*, M. Panchenko*, V. V. Zaitsev ${ }^{\ddagger}$, \\ O. V. Arkhypov ${ }^{\S}$, and H. O. Rucker*
}

\begin{abstract}
The paper deals with interpretation of long-periodic (minutes, e.g. $<0.01 \mathrm{~Hz}$ ) modulations, detected in the microwave records during flaring events on the Sun, as the signatures of large scale transverse oscillations of coronal loops, observed at the same time by TRACE. In the case of transverse large scale oscillatory motions of a loop a properly located observer, besides of the modulation caused by motion of the emission diagram pattern at the main oscillation frequency, may see also a modulation at double frequency of the loop oscillation, resulted by a varying magnetic field during each inclination of the loop, as well as weaker higher harmonics. Identification of such "modulation pairs" in the dynamic spectra of solar microwave emission and their association with the observed oscillating coronal loops form the major result of the undertaken investigation. Three different events with the detected by TRACE post-flare oscillating loops were considered in that study (Mar.23,2000; Sep.15,2001; Sep.07,2001).
\end{abstract}

\section{Introduction}

Energetic phenomena associated with the processes of solar flaring activity are naturally accompanied by different kinds of electromagnetic emission, covering a wide frequency band from radio waves to gamma-rays. Radiation, produced within a given plasma environment, carries an information on physical and dynamic conditions in a radiating source.

\footnotetext{
* Space Research Institute, Austrian Academy of Sciences, Schmiedlstrasse 6, A-8042 Graz, Austria

† Lobachevsky State University of Nizhni Novgorod, 23 Prospekt Gagarina, 603950, Nizhni Novgorod, Russia

$\ddagger$ Institute of Applied Physics, Russian Academy of Science, 46 Ul'yanov Street, 603950, Nizhni Novgorod, Russia

§ Institute of Radio Astronomy, Ukrainian Academy of Sciences, Chervonopraporna 4, 61002, Kharkiv, Ukraine
} 
This causes an exceptional importance of the electromagnetic radiation, as a diagnostic tool, for understanding the origin and physics of various dynamic phenomena on the Sun. As a relatively new, in that context, direction of study in the traditional branch of the solar microwave radio astronomy appears the analysis of long-periodic (e.g., > $1 \mathrm{~s}$ ) fluctuations of the radiation intensity [Zaitsev et al., 2003; Khodachenko et al., 2005].

Microwave radiation (hereafter, mm-radiation) from magnetic loops in solar active regions (e.g., during solar flares) is usually interpreted as a gyro-synchrotron radiation, produced by fast electrons on harmonics of the gyro-frequency $\nu_{B}$ in the magnetic field $B$ of the loop. In the case of a power-law distribution of electrons in energy $f(\varepsilon) \propto \varepsilon^{-\delta}$, the intensity of gyro-synchrotron radiation $I_{\nu}$ from an optically thin loop [Dulk, 1985] is

$$
I_{\nu} \propto B^{-0.22+0.9 \delta}(\sin \theta)^{-0.43+0.65 \delta}
$$

For the observed typical values of electron energy spectrum index $2 \leq \delta \leq 7$ this gives proportionality of the intensity to a certain power of the background magnetic field and essential anisotropy of the radiation: $I_{\nu} \propto B^{1.58 \pm 6.08}(\sin \theta)^{0.87 \pm 4.12}$.

It follows from the equation (1) that variations of the loop magnetic field, associated with disturbances of the electric current in a radiating source, should modulate the intensity of the mm-radiation of the loop [Zaitsev et al., 2003; Khodachenko et al., 2005]. Another possible origin for the intensity modulation of the observed mm-radiation, followed from (1), is due to the quasi-periodic motion (oscillation) of a coronal magnetic loop, containing the radiation source. This mechanism is connected with the anisotropy of the gyrosynchrotron emission, as well as with the variation of the magnetic field value during the oscillatory motion of the loop, which both, according to the equation (1), may result in a quasi-periodic modulation of the received signal. Therefore, the analysis of long-periodic modulations of solar mm-radiation may be used for diagnostics of oscillating electric currents in the coronal loops, as well as for investigation of large scale motion of the loops (including loop oscillations). By this, it is natural to expect that structural complexity of solar active regions will be manifested in peculiarities of the emitted radiation.

In several cases, long-periodic modulations of solar mm-radiation (with multi-track spectra and certain modulation frequency drift) were interpreted as signatures of oscillating electric currents, running within the circuits of moving relative each other inductively connected coronal magnetic loops [Khodachenko et al., 2009]. By this, the dynamics of electric currents has been described by means of the equivalent electric circuit (LCRcircuit) models of the coronal loops [Zaitsev et al., 1998; Khodachenko et al., 2009]. In that respect Khodachenko et al. [2009] pointed that the frequency of long-periodic LCRoscillations of the electric current which depends on specific parameters of a coronal loop, usually stays within the interval $\nu_{L C R} \approx(0.03 \pm 1) \mathrm{Hz}$. At the same time, modulations caused by oscillatory motions of loops, containing the radiation sources, due to their direct connection with the large scale dynamics of loops, have to have the typical frequencies $<0.01 \mathrm{~Hz}$ and exhibit no drift. In view of that it has been proposed in Khodachenko et al. [2009] to distinguish, when speaking about different kinds of long-periodic modulations of the solar mm-radiation, between the low-frequency $(L F)(\approx 0.03 \pm 1 \mathrm{~Hz})$ and very-low-frequency $(V L F)(<0.01 \mathrm{~Hz})$ modulations, assuming the first to be connected with the LCR-oscillations of electric currents in the coronal loops and the second to be 
caused by large scale motions of the radiation sources together with the loops. LF modulations (e.g., $0.03 \pm 1 \mathrm{~Hz}$ ) have been studied and interpreted in terms of the equivalent electric circuit models of coronal loops in Zaitsev et al. [1998, 2003] and Khodachenko et al. [2005, 2009]. Therefore, they are not considered here. The present paper addresses the VLF modulations $(<0.01 \mathrm{~Hz})$ of solar mm-radiation and their possible relation to the large scale dynamics of coronal loops in solar active regions.

\section{Diagnostics of Oscillating Coronal Loops via VLF Modula- tions of mm-Radiation}

Large scale transverse oscillatory motion of a coronal loop is accompanied by periodic stress of magnetic field, created in the loop during each inclination, i.e. two times per the oscillation cycle. This means that the value of magnetic field fluctuates during oscillatory motion of the loop with a half-period $P_{\text {osc }} / 2$ of the loop oscillation. Therefore, according to equation (1), in the case of transverse oscillating loop, a properly located observer, besides of the modulation caused by the emission diagram motion at the main oscillation frequency $\nu_{0}=1 / P_{\text {osc }}$, may see in some cases also the modulation at the double frequency of the loop oscillation $2 \nu_{0}$, as well as weak higher order harmonics caused by the nonlinearity of Equation (1). Domination of the main and double-frequency harmonics in the spectrum is due to the fact that these frequencies, according to the above mentioned specifics of the intensity modulating factors, are implicitly present in the signal. In the most cases of real observed signals only these first two harmonics can be detected. Due to the same reason it is difficult to expect appearance of significant (clearly detectable) harmonics beyond the main frequency, caused only by the nonlinearity of Equation (1), when the modulation of mm-radiation is connected only with the varying magnetic field, as it takes place in the case of LCR-oscillations of electric current in the loop [Zaitsev et al., 1998, 2003; Khodachenko et al., 2005], or for any internal wave process (e.g., sausage mode). Therefore, the presence of "modulation pairs" in the spectra, i.e., the lines which may be associated with a main and double frequencies of a loop oscillation $\left(\nu_{0}\right.$ and $\left.2 \nu_{0}\right)$ could indicate about a transverse oscillatory dynamics of the loop.

VLF modulations in solar microwave bursts recorded in Metsähovi Radio Observatory (Finland) with the $14-\mathrm{m}$ and $1.8 \mathrm{~m}$ radio telescope antenna at $37 \mathrm{GHz}$ and $11.7 \mathrm{GHz}$, respectively are considered here. By this, the key selection criterion for the analyzed microwave data was their synchronism with the oscillating loops observed in EUV by TRACE. At $37 \mathrm{GHz}$ the Metsähovi radio telescope is able to resolve spatially an active region with oscillating loops. This enables analysis of mm-radiation emitted directly from the region, imaged in EUV (e.g observed by TRACE). At $11.7 \mathrm{GHz}$ the analyzed radio signal is collected from the whole solar disk. However, also in this case, by comparison with observations in other wavelengths and timing of the events, it is always possible to identify modulation features related to energy release and dynamic phenomena in particular active regions.

A combination of the sliding window Fourier (SWF) transform and the nonlinear WignerVille (WV) method [Cohen, 1989], has been applied for detection of VLF quasi-periodic 
features in the records of the solar mm-radiation intensity. Both methods (WV and SWF), combined in a proper way, are realized as a common algorithm [Zaitsev et al., 1998], together with different types of signal processing and filtration. Among the advantages of the combined SWF-WV data analysis algorithm are its high sensitivity, high spectral and temporal resolution, as well as the ability to detect complex multi-signal modulations. The outcome of the SWF-WV algorithm appears in the form of dynamic spectra of the VLF signals, modulating the radiation. Besides of that, for visualization of the whole variety of detected modulations, averaged spectral density plots are used. These plots are obtained by averaging of multiple instantaneous cuts of the dynamic spectrum, so that also the temporarily existing modulation features become visible among the longer lasting modulation lines. SWF-WV data analysis algorithm is now available to scientific community via the on-line catalogue of models and data analysis tools ${ }^{1}$, developed within the JRA3-EMDAF (European modelling and data analysis facilities) activity ${ }^{2}$ of the research infrastructure project Europlanet-RI.

Among the available at Metsähovi solar microwave emission records three events coincide in time with the observed by TRACE large scale transverse oscillations of coronal loops. These events are considered below.

Figure 1a shows the dynamic spectrum and average spectral density plot of VLF modulations in the mm-radiation from the active region AR8910 where a group of oscillating loops was observed after M2.0 flare on 2000-Mar-23, at 11:30-12:00 UT [Aschwanden et al., 2002; Schrijver et al., 2002]. These observations were performed at $37 \mathrm{GHz}$, and the analyzed radiating source in the active region AR8910 was well resolved by the Metsähovi radio telescope. A remarkable feature of the VLF modulation spectrum in Fig. 1a consists in the presence of "modulation pairs", i.e., modulations at a given and double frequency: (a) at $1.7 \mathrm{mHz}(\sim 10 \mathrm{~min})$ and $3.4 \mathrm{mHz}(\sim 4.9 \mathrm{~min})$; (b) at $6.0 \mathrm{mHz}(\sim 2.8 \mathrm{~min})$ and $12.0 \mathrm{mHz}(\sim 1.4 \mathrm{~min})$, as well as $(\mathrm{c})$ at $7.8 \mathrm{mHz}(\sim 2.1 \mathrm{~min})$ and $15.6 \mathrm{mHz}(\sim 64 \mathrm{~s})$. These "modulation pairs" could indicate the transverse oscillating loops with the periods, corresponding to the main frequencies of pairs, i.e. $\sim 10 \mathrm{~min} ; \sim 2.8 \mathrm{~min}$, and $\sim 2.1 \mathrm{~min}$ for the cases (a), (b), and (c), respectively. By this, the first "modulation pair" (case (a)) as a signature of the loop transverse oscillation with a period $\sim 10 \mathrm{~min}$, fits very well the results of TRACE observations, which registered the oscillating loop with approximately the same period ( $\sim 615 \mathrm{~s}$ ) [Aschwanden et al., 2002; Schrijver et al., 2002]. As it can be seen from the dynamical spectrum in Figure 1a, the spectral resolution of performed analysis in this particular case was $\sim 0.3 \mathrm{mHz}$. By this, the frequency $1.62 \mathrm{mHz}$ which corresponds the detected with TRACE period $615 \mathrm{~s}$ definitely stays within the frequency interval $1.7 \pm 0.3 \mathrm{mHz}$ of the corresponding modulation feature revealed by the analysis of mm-radiation. It makes no sense to search in TRACE data the signatures of other oscillating loops (cases (b) and (c)), indicated by the VLF modulations of mm-radiation during 2000-Mar-23 event, since with the usual $40 \mathrm{~s}$ image sampling cadence of TRACE and the 4-point resolution limit of the instrument [Aschwanden et al., 2002] the fastest detectable by TRACE period is about $3 \mathrm{~min}$. The remaining short-periodic "non-paired" modulation feature at $8.4 \mathrm{mHz}(\sim 1.9 \mathrm{~min})$ may be a signature of an other oscillatory process, not connected with large scale transverse motion of loops, e.g. a sausage-type

\footnotetext{
$\overline{{ }^{1} \text { http://europlanet-jra3.oeaw.ac.at/catalogue/ }}$

${ }^{2}$ http://europlanet-jra3.oeaw.ac.at/
} 

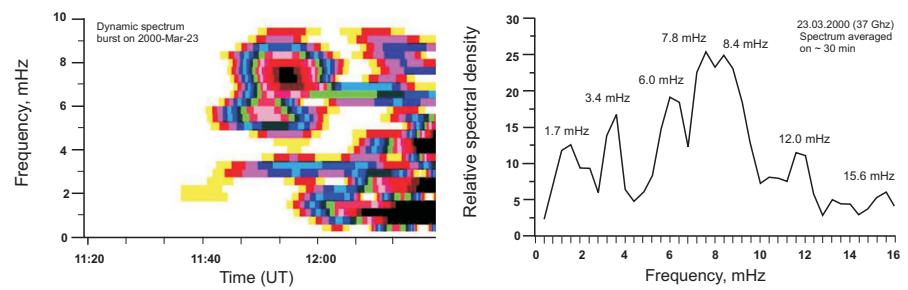

a
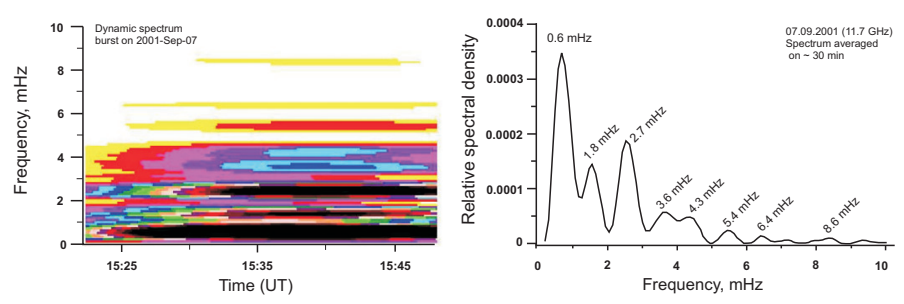

b
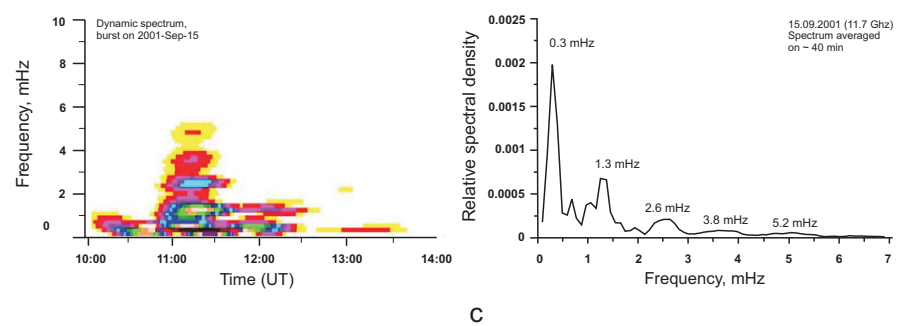

Figure 1: Dynamic spectra (left) and average spectral density plots (right) of very-low-frequency modulations of solar microwave bursts on (a) 2000-Mar-23 (11:20-12:20 UT); (b) 2001-Sep-07 (15:20-15:50 UT); (c) 2001-Sep-15 (10:00-14:00 UT).

MHD wave excited in a loop. Detailed analysis of this special case stays however beyond the scope of the present short publication.

The burst on 2001-Sep-07 represents another example of possible manifestation of transverse oscillating coronal loops in microwaves. The burst was produced during M-flare activity at 15:30 UT in the active region AR9601, where TRACE have registered also a group of oscillating loops, observed immediately after the flare [Aschwanden et al., 2002]. The corresponding mm-radiation record was made at $11.7 \mathrm{GHz}$. Thus, the emission from the whole solar disk contributed to the analyzed microwave intensity profile. At the same time, as can be seen in the VLF modulation dynamic spectrum in Fig. 1b, the most of modulation lines begin simultaneously with the impulsive phase of the flare in the active region AR9601 at 15:30 UT and can be therefore associated with this event. Three "modulation pairs" can be identified in the dynamic and averaged spectra in Fig. 1b: (a) $1.8 \mathrm{mHz}(\sim 9.2 \mathrm{~min})$ and $3.6 \mathrm{mHz}(\sim 4.6 \mathrm{~min})$; (b) $2.7 \mathrm{mHz}(\sim 6.2 \mathrm{~min})$ and 5.4 $\mathrm{mHz}(\sim 3.1 \mathrm{~min}) ;$ (c) $4.3 \mathrm{mHz}(\sim 3.8 \mathrm{~min})$ and $8.6 \mathrm{mHz}(\sim 1.9 \mathrm{~min})$, which may be 
the signatures of transverse oscillating loops with the periods $\sim 9.2 \mathrm{~min}, \sim 6.2 \mathrm{~min}$, and $\sim 3.8 \mathrm{~min}$, respectively. We would like to note in that respect that the loop periods in the cases (a) and (b) again are consistent with the detected by TRACE 6-10 min oscillating loops [Aschwanden et al., 2002], whereas the shorter period oscillation (case (c)) can not be resolved by TRACE due to the relatively long image sampling cadence. The modulation at $5.4 \mathrm{mHz}(\sim 3.1 \mathrm{~min})$ may be also a weak third harmonic produced by the $9.2 \mathrm{~min}$ oscillating loop. If this is true, then the line at $2.7 \mathrm{mHz}(\sim 6.2 \mathrm{~min})$ will have no a pair-companion, and one should exclude the possibility of the $\sim 6.2$ min transverse oscillating loop. A "non-paired" weak modulation feature at $6.4 \mathrm{mHz}(\sim 2.6 \mathrm{~min})$ may be a signature of an oscillating loop with not resolved second harmonic. At the same time, similar to the case of 2000-Mar-23 event, weak short-periodic harmonics may be also a feature of oscillatory processes, not connected with the transverse motion of a loop, but resulted by a changing magnetic field only. The strong modulation line at $0.6 \mathrm{mHz}(\sim 27.7$ $\mathrm{min}$ ) in the spectra of 2001-Sep-07 event has to be considered separately from all other modulations mentioned above. The dynamical spectrum in Fig. 1b, as well as a separate study of VLF modulations of the mm-radiation recorded before the faring burst at 15:30 UT in the active region AR9601, indicate the presence of the $\sim 27.7 \mathrm{~min}$ component also before the flare. Since the analyzed microwave emission was received from the whole solar disk, the $\sim 27.7$ min modulation feature originates very likely in another active region. It may be also connected with a kind of global solar seismology process.

The last available example of microwave observations of transverse oscillating coronal loops is the event on 2001-Sep-15, associated with M flare at 11:23 UT in the active region AR9608. The microwave burst on 2001-Sep-15 was observed at $11.7 \mathrm{GHz}$, and the mm-radiation source in AR9608 was not resolved by the Metsähovi antenna. However, similar to the case of burst on 2001-Sep-07 all the spectral features related to the flaring active region AR9608 can be identified by event timing. In particular, most of the detected modulation lines begin simultaneously with the impulsive phase of the flare at 11:23 UT (see Fig. 1c). At least one "modulation pair": $1.3 \mathrm{mHz}(\sim 12.8 \mathrm{~min})$ and $2.6 \mathrm{mHz}(\sim 6.4$ min) can be identified in the modulation spectra in Fig. 1c. It may be connected with $\mathrm{a} \sim 12.8 \mathrm{~min}$ transverse oscillating loop. This result agrees with the reported TRACE observations of oscillating loops in the active region AR9608 with a period $\sim 12-15 \mathrm{~min}$ [Verwichte et al., 2010]. Other, more short-periodic modulations at $3.8 \mathrm{mHz}(\sim 4.4 \mathrm{~min})$ and $5.2 \mathrm{mHz}(\sim 3.2 \mathrm{~min})$ could be the higher order harmonics, produced by the $12.8 \mathrm{~min}$ oscillating loop, or the modulations associated with oscillatory processes not connected with transverse motion of loops. A special remark deserves the ultra-low-frequency (ULF) modulation at $0.3 \mathrm{mHz}(\sim 56 \mathrm{~min})$ in the 2001-Sep-15 spectra in Figs. 1c. This ULF modulation appears before the flaring burst (at 11:23 UT) and lasts much longer than all other modulation lines in the spectrum. Therefore it cannot be related to the flare in the active region AR9608. This modulation is probably connected with the solar seismology processes, or a slow dynamics of another active region.

As it can be seen from the dynamic spectra in Fig. 1, the available microwave emission records do not cover up to the end the events on 2000-Mar-23 and 2001-Sep-7, whereas the event on 2001-Sep-15 is covered rather well. In particular, the main component of the modulation pair at $1.3 \mathrm{mHz}$ (e.g., $T \sim 12.8 \mathrm{~min}$ ) is clearly seen to decay at the interval $\tau$ of about 90 minutes. This means that the quality factor $Q=\pi \tau / T$ of this oscillation 
is about 22. Similar values of $Q$ factor may be expected also for the loop oscillations detected on 2000-Mar-23 and 2001-Sep-7. In both cases the oscillations show the trend of having a decay time of at least $5-7$ periods. This corresponds to $Q$ factor values $15-22$.

\section{Magnetic Field Variations, Estimated from VLF Spectra}

Unfortunately, using the available data, it is impossible to perform an exact calculation for the amplitude of magnetic field variations. This is because the analyzed microwave signals were recorded in relative units without calibration to the radiation intensity scale. At the same time, taking into account known values of the maximal intensity measured during the radio bursts and the lower limit of sensitivity of the Metsähovi receiver, the intensity modulation amplitude $\Delta I_{\nu}$ can be estimated very roughly from the obtained VLF spectra, by their comparison with the spectra of specially created modelling signals [Khodachenko et al., 2005]. For the considered in the paper events this estimation gives the value of the relative variation of intensity $\Delta I_{\nu} / I_{\nu}^{0} \sim 10^{-2} \pm 10^{-1}$. Assuming, as an upper rough limit, that this variation of intensity is connected only with the varying magnetic field, we come to a relation $\Delta I_{\nu} / I_{\nu}^{0}=\left(B / B_{0}\right)^{\gamma}-1=\left(\left(\Delta B / B_{0}+1\right)^{\gamma}-1\right)$, where $\Delta B / B_{0}$ is the relative variation of magnetic field, and $\gamma=-0.22+0.9 \delta$ is the power index of magnetic field in the equation (1). For $2<\delta<7$ one gets that $\gamma=1.58 \pm 6.08$. For the case of small relative variations of the magnetic field we finally obtain $\Delta I_{\nu} / I_{\nu}^{0} \approx \gamma \Delta B / B_{0}=10^{-2} \pm 10^{-1}$, which for $B_{0}=100 \mathrm{G}$ gives the amplitude $\Delta B=(0.16 \pm 6.3) \mathrm{G}$.

In view of this estimation we would like to note that the second harmonic caused only by to the nonlinearity of Equation (1) should be rather weak $\left(\sim \Delta B / B_{0}\right.$ of the main harmonic). The detected higher amplitudes of the second harmonic indicate about the fact that the "modulation pairs" originate due to transverse oscillatory motion of coronal loops, so that the modulation mechanism includes also motion of the radiation source together with the oscillating loop. The weak second harmonic caused by the non-linearity of Equation (1) in the case of a varying in time B only, is in particular, the reason that only the major frequency features were detected in the observations of modulation lines associated with oscillating electric currents (e.g., magnetic field) in the electric circuits of coronal loops [Khodachenko et al., 2009].

\section{Conclusions}

The aim of this publication was to show that in several (available) cases VLF modulations of solar mm-radiation have similar temporal behaviour and coincide in time with the decaying large scale transverse oscillatory motions of the coronal loops triggered by flares. Such a coincidence indicates about a possible physical link between the oscillatory motion of the loops and temporal variations of the recorded radio emission. As a working hypothesis to take this link into account, a loop with propagating beams of non-thermal particles, generating microwave emission by electron gyro-synchrotron mechanism, has been considered. As it has been pointed in Schrijver et al. [2002], who studied several cases of observed with TRACE transverse oscillations in coronal loops, including the event 
on 2000-Mar-23 addressed in the present paper, in almost all cases the oscillating loops lie at or near large scale separatrices or near sites involved in reconnection. These regions may be the sources of non-thermal particles injected into the loops.

Joint action of two radiation modulating factors: (i) the quasi-periodic fluctuation of magnetic field and (ii) motion of the radio emission diagram, in the case of a transverse oscillating coronal loop results in essentially non-sinusoidal (non-harmonic) character of the signal received by a remote observer, with strongly pronounced two first harmonics (at the main and double frequency of the oscillation), called in the present paper as "modulation pairs". Such specifics of the VLF spectrum has been used for the identification of oscillating loops triggered by flares. Comparison of parameters of the "modulation pairs" with the simultaneous TRACE observations in EUV enabled to associate some of the paired VLF modulation features, especially those having the main period of $6 \pm 15$ min, with the large scale transverse oscillations of coronal loops. The shorter-period "modulation pairs" with the main period $<3$ min may be also connected with transverse oscillating post-flare loops, however, these loops could not be resolved by the TRACE instrument. The "non-paired" features in the VLF modulation dynamic spectra, detected sometimes, may be either parts of "modulation pairs", in which the second harmonic cannot be resolved due to strong contamination of the analyzed signal, or be the features of other oscillatory processes (MHD modes) in the loops, not connected with their large scale transverse motion, e.g., sausage-type MHD waves. Investigation of this opportunity deserves a special study which appears beyond the scope of the present publication.

Exact detection of transverse motion of coronal loops by the dynamical spectra of microwave emission (with exclusion of other mechanisms which may also generate higher spectral harmonics) requires a quantitative study of the measured radio signal and superimposing these results with precise calculation of radiation from an oscillating loop, taking into account the loop position relative observer. This should involve dedicated numerical modelling, opening a special direction for further development of the ideas expressed in the paper.

Acknowledgments. This work was supported by the Austrian Fonds zur Förderung der wissenschaftlichen Forschung (project P21197-N16). T.V.Z. also thanks the grant GNSF/ST06/4-098 of Georgian National Science Foundation. O.V.A. thanks Academic Exchange program of the Austrian Academy of Sciences and the project "Chadra" (No. 0107U001024) of National Academy of Sciences of Ukraine.

\section{References}

Aschwanden, M. J., B. DePontieu, C. J. Schrijver, and A. Title, Transverse Oscillations in Coronal Loops Observed with TRACE: II. Measurements of Geometric and Physical Parameters, Solar Phys., 206, 99-132, 2002.

Cohen, L., Time-frequency distributions - A review, IEEE Proc., 77, 941-981, 1989.

Dulk, G. A., Radio emission from the sun and stars, Ann. Rev. Astron. Astrophys., 23, 169-224, 1985. 
Khodachenko, M. L., V.V. Zaitsev, A. G. Kislaykov, H. O. Rucker, and S. Urpo, Lowfrequency modulation in the solar microwave radiation as a possible indicator of inductive interaction of coronal magnetic loops, Astron. Astrophys., 433, 691-699, 2005 .

Khodachenko, M. L., V. V. Zaitsev, A. G. Kislyakov, and A. V. Stepanov, Equivalent electric circuit models of coronal magnetic loops and related oscillatory phenomena on the Sun, Space Sci. Rev., 149, 83-117, 2009.

Schrijver, C.J., M. J. Aschwanden, and A. M. Title, Transverse oscillations in coronal loops observed with TRACE I. An Overview of Events, Movies, and a Discussion of Common Properties and Required Conditions, Solar Phys., 206, 69-98, 2002.

Verwichte E., C. Foullon, and T. Van Doorsselaere, Spatial Seismology of a Large Coronal Loop Arcade from TRACE and EIT Observations of its Transverse Oscillations, Astrophys. J., 717, 458-467, 2010.

Zaitsev, V. V., A. V. Stepanov, S. Urpo, and S. Pohjolainen, LRC-circuit analog of currentcarrying magnetic loop: diagnostics of electric parameters, Astron. Astrophys., 337, 887-896, 1998.

Zaitsev, V.V., A. G. Kislyakov, S. Urpo, A. V. Stepanov, and E. I. Shkelev, SpectralTemporal Evolution of Low-Frequency Pulsations in the Microwave Radiation of Solar Flares, Astron. Rep., 47, 873-882, 2003. 
